Pacific Journal of Mathematic 


\title{
MAPPING PROPERTIES OF CESÀRO SUMS OF ORDER TWO OF THE GEOMETRIC SERIES
}

\author{
S. E. RAUCH
}

1. Introduction. Previous investigations of the mappings

$$
w=S_{n}^{(k)}(z)
$$

of the unit circle $|z| \leq 1$, where

$$
S_{n}^{(k)}(z)=\left(\begin{array}{c}
n+k \\
k
\end{array}\right)+\left(\begin{array}{c}
n+k-1 \\
k
\end{array}\right) z+\cdots+\left(\begin{array}{l}
k \\
k
\end{array}\right) z^{n}
$$

denotes the $n$th Cesàro sum of order $k$ of the geometric series, have been made by Fejer, Schweitzer, Sidon, and Szegö. Knowledge of the properties of the sums $S_{n}^{(k)}(z)$ is valuable in the study of power series having coefficients monotonic of order $k+1$.

The present article provides additional asymptotic properties for

$$
S_{n}^{(2)}\left(e^{i \phi}\right)=x_{n}(\phi)+i y_{n}(\phi) \text {. }
$$

The following results are established:

THEOREM 1. For $n$ sufficiently large, an $\alpha_{n}$ exists such that $y_{n}(\phi)$ is increasing for $0<\phi<\alpha_{n}$ and decreasing for $\alpha_{n}<\phi<\pi$. Furthermore,

$$
\alpha_{n}=\alpha / n+O\left(n^{-2}\right) \text {, where } \pi<\alpha<3 \pi / 2 \text {. }
$$

THEOREM 2. For $n$ sufficiently large, a $\beta_{n}$ exists such that

$$
x_{n}^{\prime}(\phi)\left\{\begin{array}{l}
\leq 0,0<\phi<\beta_{n}, n \equiv 0(\bmod 3) \\
<0,0<\phi<\beta_{n}, n \equiv 1(\bmod 3) \\
<0,0<\phi<\beta_{n}, n \equiv 2(\bmod 3)
\end{array}\right.
$$

Received January 28, 1953.

Pacific J. Math. 4 (1954), 109-121 
where

$$
\beta_{n}=\frac{2 \pi}{3}+\frac{\beta}{n}-O\left(n^{-3 / 2}\right),
$$

and $\beta=2 \pi, 4 \pi / 3,2 \pi / 3$ for $n \equiv 0,1,2(\bmod 3)$, respectively.

THEOREM 3. For $n$ sufficiently large, the mapping of $|z|=1$ by

$$
w=S_{n}^{(2)}\left(e^{i \phi}\right)
$$

is convex for $0<\phi<\gamma_{n}$, where $\gamma_{n}$ is the maximum angle for which convexity holds, and $\gamma_{n}=\gamma / n+O\left(n^{-2}\right)$ where $2 \pi<\gamma<3 \pi$.

\section{Proof of Theorem 1.}

2.1. A closed expression for $y_{n}^{\prime}(\phi)$ has been presented by Szegö [10]:

$$
\begin{aligned}
& y_{n}^{\prime}(\phi)= \\
& \frac{1}{8 \sin ^{2} \phi / 2}\left\{-\left(n^{2}+3 n+3\right)-n \cdot \frac{\sin (n+3 / 2) \phi}{\sin \phi / 2}+3 \frac{\sin ^{2}(n+1) \phi / 2}{\sin ^{2} \phi / 2}\right\}
\end{aligned}
$$

The inequality $y_{n}^{\prime}(\phi)<0$ is satisfied if

$$
n^{2}+3 n+3>-n \cdot \frac{\sin (n+3 / 2) \phi}{\sin \phi / 2}+3 \frac{\sin ^{2}(n+1) \phi / 2}{\sin ^{2} \phi / 2},
$$

or

$$
n^{2}+3 n+3>n \cdot \csc \phi / 2+3 \cdot \csc ^{2} \phi / 2 \text {. }
$$

Let $\delta$ be fixed, $\delta>0$, and consider the restriction $\phi>\delta / n$. For $n$ sufficiently large, $\sin (\delta / 2 n)>\delta / \pi n$, and the previous inequality is maintained if $\delta$ is chosen so that

$$
n^{2}+3 n+3>n^{2} \pi / \delta+3 \pi^{2} n^{2} / \delta^{2} \text {, or } \pi / \delta+3 \pi^{2} / \delta^{2}<1 \text {. }
$$

It is sufficient for the present problem to define $\delta=3 \pi$. Hence, if $\phi \geq 3 \pi / n$, then $y_{n}^{\prime}(\phi)<0$ and $3 \pi / n>\alpha_{n}$. Since 


$$
y_{n}^{\prime}(\phi)=\sum_{m=1}^{n} a_{m} \cos m \phi
$$

where

$$
a_{m}=m\left(\begin{array}{c}
n+2-m \\
2
\end{array}\right)
$$

it at once follows that $y_{n}^{\prime}(\phi)>0$ for $0 \leq \phi \leq \pi / 2 n$.

2.2. In the next section it is shown that in the interval $\pi / 2 n<\phi<3 \pi / n$ there is exactly one $\phi=\alpha_{n}$ such that $y_{n}^{\prime}(\phi)=0$ if $n$ is sufficiently large. More precisely, for the $\phi=\alpha_{n}$ the second derivative does not vanish and $\alpha_{n} \sim \alpha / n$, $\alpha>0$, where $\pi<\alpha<3 \pi / 2$. The magnitude of $\alpha$ is defined as the root of a transcedental equation.

It is possible to express $(2.1)$ in the following form:

$$
y_{n}^{\prime}(\phi)=n^{2} / 8 \cdot g_{n}(\phi) \cdot \csc ^{2} \phi / 2,
$$

where the function $g_{n}(\phi)$ is defined as

$$
g_{n}(\phi)=-1-\frac{1}{n} \cdot \frac{\sin (n+3 / 2) \phi}{\sin \phi / 2}+\frac{3}{n^{2}} \cdot \frac{\sin ^{2}(n+1) \phi / 2}{\sin ^{2} \phi / 2}-\left(\frac{3}{n}+\frac{3}{n^{2}}\right)
$$

Let $\phi=c / n, \pi / 2<c<3 \pi$, and then $g_{n}(\phi)$ becomes a function of $c$, denoted by $G_{n}(c)$. In addition,

$$
\lim _{n \rightarrow \infty} G_{n}(c)=-1 / c^{2} \cdot f(c)
$$

where

$$
f(c)=2 c \cdot \sin c+6 \cdot \cos c+\left(c^{2}-6\right) \text {. }
$$

Furthermore, $G_{n}(c)$ converges uniformly to this limit for arbitrary values of $c$ in the interval. It is sufficient to show that the function $f(c)$ has a unique simple zero in the interval $\pi / 2<c<3 \pi / 2$ to assure that $g_{n}(c)$ has a simple zero in the same interval if $n$ is sufficiently large.

An easy calculàtion yields

$$
f^{\prime}(c)=8 \cdot \cos ^{2}(c / 2) \cdot(c / 2-\tan c / 2) .
$$


Thus it is seen that $f(0)=0, f^{\prime}(c)<0$ for $0<c<\pi ; f(\pi)=-\left(12-\pi^{2}\right)$ $<0, f^{\prime}(c)>0$ for $\pi<c<2 \pi ; f(2 \pi)>0$ and if $c>2 \pi$ then $f(c)>(c-1)^{2}$ $-13>0$. Since $f(3 \pi / 2)>0$, there is a simple positive zero, $c=\alpha$, of the function $f(c), \pi<\alpha<3 \pi / 2$. In conclusion, $\alpha_{n} \sim \alpha / n, \pi<\alpha<3 \pi / 2$, for $n$ sufficiently large.

2.3. It is not difficult to find a more precise asymptotic expression for $\alpha_{n}$. For this purpose let $\alpha_{n}=c / n$, where $c=\alpha+a / n$ and $a$ is a bounded, real constant. Let $h_{n}(a)$ denote $g_{n}(c)$ when the latter is regarded as a function of $a$. Let $\phi=c / n$; a simplification yields

$$
-n^{2} h_{n}(a)=n^{2}\left(2 c \cdot \sin c+6 \cos c+c^{2}-6\right) / c^{2}+n(3+3 \cos c-6 / c \cdot \sin c)
$$

$$
+(5 / 2-13 c / 6 \cdot \sin c-5 / 2 \cdot \cos c)+O(1 / n)
$$

If

$$
h(c)=3 c^{2}[1+\cos c-2 / c \cdot \sin c]
$$

and

$$
k(c)=c^{2}[5 / 2-13 c / 6 \cdot \sin c-5 / 2 \cdot \cos c],
$$

then it is possible to rewrite the previous expression in the form

$$
-n^{2} c^{2} h_{n}(a)=n^{2} \cdot f(c)+n \cdot h(c)+k(c)+O(1 / n) .
$$

Let the functions $f(c), h(c), k(c)$ be expanded by Taylor's formula for values of $c$ near $\alpha$. Then the previous equality becomes

$$
-n^{2} c^{2} h_{n}(a)=n\left[a \cdot f^{\prime}(\alpha)+h(\alpha)\right]+a^{2} / 2 \cdot f^{\prime \prime}(\alpha)+a \cdot h^{\prime}(\alpha)+k(\alpha)+O(1 / n) .
$$

Thus one obtains

$$
\lim _{n \rightarrow \infty}\left[-n \cdot c^{2} h_{n}(a)\right]=a \cdot f^{\prime}(\alpha)+h(\alpha) \text { and } f^{\prime}(\alpha) \neq 0
$$

Obviously the limit has a zero for the value $a=-h(\alpha) / f^{\prime}(\alpha)$, or

$$
a=-3 \alpha^{2} / 8 \cdot(1+\cos \alpha-2 / \alpha \cdot \sin \alpha) \cdot \sec ^{2} \alpha / 2 \cdot(\alpha / 2-\tan \alpha / 2)^{-1} \text {, }
$$

and $\alpha$ is the simple zero of the function

$$
f(c)=2 c \cdot \sin c+6 \cdot \cos c+c^{2}-6
$$


in the interval $\pi<\alpha<3 \pi / 2$.

This shows that for $n$ sufficiently large, $y_{n}^{\prime}(\phi)=0$ for

$$
\phi=\alpha_{n}=\alpha / n+\left(a+\epsilon_{n}\right) / n^{2},
$$

where $\epsilon_{n} \longrightarrow 0$. Thus the assertion of Theorem 1 has been verified.

\section{Proof of Theorem 2 .}

3.1. In the article by Szegö [10], a closed expression for $x_{n}^{\prime}(\phi)$ is presented:

(3.1) $\quad x_{n}^{\prime}(\phi)$

$$
=\frac{\cos \phi / 2}{8 \sin ^{3} \phi / 2}\left[-(2 n+3)-(n+3 / 2) \frac{\cos (n+3 / 2) \phi}{\cos \phi / 2}+\frac{3}{2} \cdot \frac{\sin (n+3 / 2) \phi}{\sin \phi / 2}\right] .
$$

It inmediately follows that $x_{n}^{\prime}(\phi)$ is negative if

$$
[3 /(2 n+3)]^{2} \cdot \csc ^{2} \phi / 2+\sec ^{2} \phi / 2<4 ; \cot \phi / 2>0 \text {. }
$$

Let $0<\phi \leq \pi / n$. As

$$
x_{n}(\phi)=\sum_{m=1}^{n} b_{m} \cos m \phi,
$$

where

$$
b_{m}=\left(\begin{array}{c}
n+2-m \\
2
\end{array}\right)
$$

then $x_{n}^{\prime}(\phi)<0$. Next consider the interval $\pi / n \leq \phi \leq 2 \pi / 3-c / n$, where $c$ is fixed, $c>0$. Since

$$
[3 /(2 n+3)]^{2} \cdot \csc ^{2} \phi / 2+\left[1-\sin ^{2} \phi / 2\right]^{-1},
$$

as a function of $\sin ^{2} \phi / 2$, is convex from below, it obtains its maximum at one or both end-points of the interval. Thus in order to prove the inequality (3.2) it is sufficient to consider only the end-point values of $\pi / n \leq \phi \leq 2 \pi / 3-c / n$. It easily follows that (3.2) is satisfied by $\phi=\pi / n$. Now study $\phi=2 \pi / 3-c / n$. Since 


$$
\sin ^{-2} \phi / 2=O(1), \cos ^{2} \phi / 2=1 / 4 \cdot(1+\sqrt{3} \cdot c / n)+O\left(1 / n^{2}\right),
$$

the left side of $(3.2)$ then can be written as

$$
[3 /(2 n+3)]^{2} \cdot O(1)+4\left[1+\sqrt{3} \cdot c / n+O\left(1 / n^{2}\right)\right]^{-1}
$$

$$
=4(1-\sqrt{3} \cdot c / n)+O\left(1 / n^{2}\right),
$$

which indeed is less than 4 provided $n$ is sufficiently large. The minimum value of $n$ is a function of $c$. Thus it now is established that $x_{n}^{\prime}(\phi)<0$ for $0<\phi \leq$ $2 \pi / 3-c / n$, if $n$ is sufficiently large, $n \geqq n_{1}(c)$, where $c$ is an arbitrary positive fixed magnitude.

3.2. Next let $\phi=2 \pi / 3$. By (3.1) it follows that

$$
x_{n}^{\prime}(2 \pi / 3)=-(2 n+3) / 6 \sqrt{3} \cdot(1-\cos 2 \pi n / 3)-1 / 6 \cdot \sin 2 \pi n / 3 .
$$

Three possible cases for the $n$ arise. For $n \equiv 0(\bmod 3), x_{n}^{\prime}(2 \pi / 3)=0$; whereas for $n \equiv 1,2(\bmod 3), x_{n}^{\prime}(2 \pi / 3)<0$. Thus the behavior of $x_{n}^{\prime}(\phi)$ in the neighborhood of $\phi=2 \pi / 3$ must be examined more fully, $n \equiv 0(\bmod 3)$. Let

$$
x_{n}^{\prime}(\phi)=r(\phi) \cdot s(\phi) \text {, }
$$

where

$$
r(\phi)=1 / 8 \cdot \cos \phi / 2 \cdot \csc ^{3} \phi / 2
$$

and

$$
\begin{aligned}
s(\phi)=-(2 n+3)-(n+3 / 2) \cdot \cos (n+3 / 2) \phi & \cdot \sec \phi / 2 \\
& +3 / 2 \cdot \sin (n+3 / 2) \phi \cdot \csc \phi / 2 .
\end{aligned}
$$

As $s=0$ for $\phi=2 \pi / 3$, then

$$
x_{n}^{\prime \prime}(2 \pi / 3)=r(2 \pi / 3) \cdot s^{\prime}(2 \pi / 3) .
$$

Upon letting $N=n+3 / 2$, we see that

$$
s^{\prime}(2 \pi / 3)=0, x_{n}^{\prime \prime}(2 \pi / 3)=0 \text {. }
$$

An examination of the third derivative shows that

$$
x_{n}^{\prime \prime \prime}(2 \pi / 3)=r(2 \pi / 3) \cdot s^{\prime \prime}(2 \pi / 3) \text {. }
$$


As $r(2 \pi / 3)>0, \operatorname{sgn} x_{n}^{\prime \prime}(\phi)=\operatorname{sgn} s^{\prime \prime}(\phi)$. Since

$$
s^{\prime \prime}(\phi)=N^{3} \sec \phi / 2 \cdot \cos N \phi+O\left(N^{2}\right),
$$

then $s^{\prime \prime}(2 \pi / 3)=-2 N^{3}+O\left(N^{2}\right)$, and for $n$ sufficiently large $s^{\prime \prime}(2 \pi / 3)<0$. It is now known than $x_{n}^{\prime}(\phi)<0$ for $0<\phi<2 \pi / 3$ if $n$ is sufficiently large.

3.3. This section extends the investigation beyond $\phi=2 \pi / 3$. For this pur pose let $\phi=2 \pi / 3+c / N$, where again $N=n+3 / 2$. The substitution of this value of $\phi$ into ( 3.1$)$ yields

(3.3) $\frac{8 \sin ^{3} \phi / 2}{\cos \phi / 2} \cdot x_{n}^{\prime}(\phi)=-2 N\left[1-\frac{1}{2} \frac{\cos (2 \pi n / 3+c)}{\cos \phi / 2}\right]-\frac{3}{2} \cdot \frac{(2 \pi n / 3+c)}{\sin \phi / 2}$.

Any easy calculation shows that

$$
\begin{gathered}
\sin \phi / 2=\sqrt{3} / 2+c / 4 N+c^{2} \cdot O\left(1 / N^{2}\right), \\
\cos \dot{\phi} / 2=1 / 2-\sqrt{3} \cdot c / 4 N+c^{2} \cdot O\left(1 / N^{2}\right) .
\end{gathered}
$$

The remainder of the section will study the separate cases of $n(\bmod 3)$.

$n \equiv 0(\bmod 3)$. Let us rewrite $(3.3)$ as follows:

$$
\begin{aligned}
& \frac{8 \sin ^{3} \phi / 2 \cdot x_{n}^{\prime}(\phi)}{\cos \phi / 2 \cdot 2(1-\cos c)} \\
& \quad=-N+\frac{\sqrt{3}}{2}\left[\frac{c \cdot \cos c-\sin c}{1-\cos c}\right]+\frac{c^{2}}{1-\cos c} \cdot O\left(\frac{1}{N}\right) .
\end{aligned}
$$

Let

$$
F(c)=[c \cdot \cos c-\sin c] \cdot[1-\cos c]^{-1} \cdot
$$

Since

$$
F^{\prime}(c)=\sin c \cdot[\sin c-c][1-\cos c]^{-2},
$$

it is easily seen that $F(c)$ is decreasing for $0<c<\pi$ and increasing for $\pi<c<2 \pi$. It follows that $x_{n}^{\prime}(\phi) \leq 0$ for $0<\phi \leq 2 \pi / 3+c / N$, where $\pi<c<$ $2 \pi-\epsilon, \epsilon$ a fixed positive number. Now 


$$
c=2 \pi-\delta / \sqrt{N}
$$

$\delta$ a fixed positive number for $n$ sufficiently large. Then

$$
F(c)=4 \pi / \delta^{2} \cdot N+O(1 / N),
$$

so that, for the above value of $\phi,(3.4)$ becomes

$$
\frac{4 \sin ^{3} \phi / 2 \cdot x_{n}^{\prime}(\phi)}{\cos \phi / 2 \cdot(1-\cos c)}=-N+2 \pi \sqrt{3} \cdot N / \delta^{2}+O(1)
$$

In addition,

$$
(1-\cos c)^{-1}=(1-\cos \delta / \sqrt{N})^{-1}=O(N) .
$$

Thus

$$
x_{n}^{\prime}(\phi)<0 \text { if } 2 \pi \sqrt{3} / \delta^{2}<1,
$$

and

$$
x_{n}^{\prime}(\phi)>0 \text { if } 2 \pi \sqrt{3} / \delta^{2}>1
$$

Thus

$$
\delta=(2 \pi)^{1 / 2} \cdot(3)^{1 / 4}
$$

furnishes the critical value of $\phi$. It has been shown that, for $n \equiv 0(\bmod 3)$,

$$
x_{n}^{\prime}(\phi)<0 \text { for } 0<\phi<2 \pi / 3
$$

and

$$
x_{n}^{\prime}(\phi) \leq 0 \text { for } 0<\phi<2 \pi / 3+2 \pi / N-O\left(N^{-3 / 2}\right) \text {, }
$$

for $n$ sufficiently large.

$n \equiv 1(\bmod 3)$. It is possible to rewrite $(3.3)$ so that the right side becomes

$-2 N[1-\cos (c+2 \pi / 3)]$

$$
+\sqrt{3}[c \cdot \cos (c+2 \pi / 3)-\sin (c+2 \pi / 3)]+c^{2} \cdot O(1 / N) .
$$

By reasoning as in the previous case, one finds $x_{n}^{\prime}(\phi)<0$ for $0 \leq c \leq 4 \pi / 3-\epsilon$, $\epsilon>0$, for $n$ sufficiently large. Let 


$$
c=4 \pi / 3-\delta / \sqrt{N}
$$

Then the right side of $(3.3)$ reduces to

$$
-\delta^{2}+4 \pi / \sqrt{3}+O(1 / \sqrt{N}) .
$$

Therefore

$$
x_{n}^{\prime}(\phi)<0 \text { if } \delta>2 \cdot \pi^{1 / 2} \cdot 3^{-1 / 4},
$$

and

$$
x_{n}^{\prime}(\phi)>0 \text { if } \delta<2 \cdot \pi^{1 / 2} \cdot 3^{-1 / 4},
$$

for $n$ sufficiently large. It follows that $x_{n}^{\prime}(\phi)<0$ for $0<\phi<\beta_{n}$, where

$$
\beta_{n}=2 \pi / 3+4 \pi / 3 N-O\left(N^{-3 / 2}\right),
$$

for $n$ sufficiently large.

$n \equiv 2(\bmod 3)$. In this case the right side of $(3.3)$ becomes

$-2 N[1-\cos (c+4 \pi / 3)]$

$$
+\sqrt{3}[c \cdot \cos (c+4 \pi / 3)-\sin (c+4 \pi / 3)]+O(1 / N) .
$$

It follows that $x_{n}^{\prime}(\phi)<0$ for $0 \leq c \leq 2 \pi / 3-\epsilon, \epsilon>0$, for $n$ sufficiently large. Let

$$
c=2 \pi / 3-\delta / \sqrt{N} .
$$

Then the right side of (3.3) is equivalent to

$$
-\delta^{2}+2 \pi / \sqrt{3}+O\left(N^{-1 / 2}\right) \text {. }
$$

Thus

$$
x_{n}^{\prime}(\phi)<0 \text { if } \delta>(2 \pi)^{1 / 2} \cdot 3^{1 / 4}
$$

and

$$
x_{n}^{\prime}(\phi)>0 \text { if } \delta<(2 \pi)^{1 / 2} \cdot 3^{1 / 4} \text {, }
$$


for $n$ sufficiently large. It has been shown that $x_{n}^{\prime}(\phi)<0$ for $0<\phi<\beta_{n}$, where

$$
\beta_{n}=2 \pi / 3+2 \pi / 3 N-O\left(N^{-3 / 2}\right),
$$

for $n$ sufficiently large.

If $n+3 / 2$ is substituted for $N$, then the results expressed in Theorem 2 are proved.

\section{Proof of Theorem 3.}

4.1 The Curvature of an image is defined to be

$$
1 / \rho=\left[1+R z \cdot f^{\prime \prime}(z) / f^{\prime}(z)\right] \cdot\left[\left|z \cdot f^{\prime}(z)\right|\right]^{-1} .
$$

If the point $w=f(z)$ traverses a closed, single-valued curve in a preassigned positive direction, then the curve is called convex if

$$
1+R\left[z \cdot f^{\prime \prime}(z) / f^{\prime}(z)\right]>0 .
$$

Let us examine the inequality (4.1) for the function

$$
f\left(e^{i \phi}\right)=s_{n}^{2}\left(e^{i \phi}\right)=x_{n}(\phi)+i \cdot y_{n}(\phi)
$$

if $z=e^{i \phi}$. By the employment of differentiation and elementary algebraic steps after substituting the derivatives in the left side of (4.1), one obtains

$$
1+R\left[z \cdot f^{\prime \prime}(z) / f^{\prime}(z)\right]=\left[x_{n}^{\prime} \cdot y_{n}^{\prime \prime}-x_{n}^{\prime \prime} \cdot y_{n}^{\prime}\right] \cdot\left[x_{n}^{\prime 2}+y_{n}^{\prime 2}\right]^{-1} \text {. }
$$

Thus the condition for the mapping to be convex is satisfied if

$$
x_{n}^{\prime} \cdot y_{n}^{\prime \prime}-x_{n}^{\prime \prime} \cdot y_{n}^{\prime}>0 \text {. }
$$

4.2. The next section studies the previous condition of convexity for the function $w=s_{n}^{2}(z), z=e^{i \phi}$, where $\phi=\gamma / n, \gamma>0$, for $n$ sufficiently large.

In the present case the expressions for $y_{n}^{\prime}(\gamma / n)$ and $x_{n}^{\prime}(\gamma / n)$, for which see (2.1) and (3.1), become

$$
\begin{gathered}
y_{n}^{\prime}(\gamma / n)=n^{4} / \gamma^{4}\left[-\gamma \sin \gamma-3 \cos \gamma+3-\gamma^{2} / 2+O(1 / n)\right], \\
x_{n}^{\prime}(\gamma / n)=n^{4} / \gamma^{4}[-2 \gamma-\gamma \cos \gamma+3 \sin \gamma+O(1 / n)] .
\end{gathered}
$$


Substitution of the latter expressions into ( 4.2$)$ yields directly

$$
\begin{aligned}
& (2 \sin \gamma-\gamma \cos \gamma-\gamma)(-2 \gamma-\gamma \cos \gamma+3 \sin \gamma) \\
& \quad-(-2+\gamma \sin \gamma+2 \cos \gamma)\left(-\gamma \sin \gamma-3 \cos \gamma+3-\gamma^{2} / 2\right)+O(1 / n)>0 .
\end{aligned}
$$

Further simplification of the previous inequality, which establishes the requirement for convexity of the image of $|z|=1$, leads to the convenient form

$$
\sin \gamma(\tan \gamma / 2-\gamma / 2)\left(6-\gamma^{2} / 2-3 \gamma \cot \gamma / 2\right)+O(1 / n)>0 \text {. }
$$

The remainder of the section is devoted to determining the maximum value of $\phi=\gamma / n$ which satisfies (4.3). In particular it is shown that the maximum angle $\gamma_{n}=\gamma / n$ for which the mapping of $|z|=1$ by $w=s_{n}^{2}(z)$ is convex, where $z=e^{i \phi}$, is determined by $2 \pi<\gamma<3 \pi$, for $n$ sufficiently large.

4.3. Consider the elementary function

$$
v(\gamma)=\sin \gamma[\tan (\gamma / 2)-\gamma / 2] .
$$

Define $\gamma_{0}$ by the equality $\tan \left(\gamma_{0} / 2\right)=\gamma_{0} / 2$. Then it is easily shown that

$$
v(\gamma)\left\{\begin{array}{l}
>0,0<\gamma<2 \pi, \\
<0,2 \pi<\gamma<\gamma_{0}, \\
>0, \gamma_{0}<\gamma<3 \pi,
\end{array}\right.
$$

Let us define

$$
f(\gamma)=6-\gamma^{2} / 2-3 \gamma \cot (\gamma / 2) .
$$

Then the image of $|z|=1$ is convex if

$$
f(\gamma)\left\{\begin{array}{l}
>0,0<\gamma<2 \pi, \\
<0,2 \pi<\gamma<\gamma_{0}, \\
>0, \gamma_{0}<\gamma<3 \pi,
\end{array}\right.
$$

for $n$ sufficiently large.

Next it is shown that the first two inequalities for $f(\gamma)$ in (4.5) are satisfied, however, for $\gamma_{0}<\gamma<3 \rho$, one finds that $f(\gamma)<0$. Since

$$
f^{\prime}(\gamma) \cdot \sin ^{2}(\gamma / 2)=-\gamma \sin ^{2}(\gamma / 2)-3 / 2 \cdot \sin \gamma+3 \gamma / 2,
$$


by a further differentiation with respect to $\gamma$ one can obtain

$$
\begin{aligned}
d / d \gamma\left\{f^{\prime}(\gamma) \cdot \sin ^{2}(\gamma / 2)\right\} & =1-\cos \gamma-\gamma / 2 \sin \gamma, \\
& =\sin \gamma\{\tan (\gamma / 2)-\gamma / 2\}=v(\gamma) .
\end{aligned}
$$

Consider the interval $0<\gamma<2 \pi$. By (4.4), $v(\gamma)>0$. Also $f^{\prime}(\gamma) \cdot \sin ^{2}(\gamma / 2)=$ 0 if $\gamma=0$. Thus $f^{\prime}(\gamma) \sin ^{2}(\gamma / 2)>0$ for $0<\gamma<2 \pi$, and consequently $f^{\prime}(\gamma)>0$ for the same interval. Finally,

$$
f(0)=\lim _{\gamma \rightarrow 0^{+}} f(\gamma)=0,
$$

which establishes the fact that $f(\gamma)>0$ in the interval $0<\gamma<2 \pi$.

In the interval $2 \pi<\gamma<\gamma_{0}, v(\gamma)<0$; therefore the function $f^{\prime}(\gamma) \sin ^{2}(\gamma / 2)$ is decreasing. It follows that

$$
f^{\prime}\left(\gamma_{0}\right) \cdot \sin ^{2}\left(\gamma_{0} / 2\right)=\gamma_{0}\left[3 / 2-\sin ^{2}\left(\gamma_{0} / 2\right)\right]-3 / 2 \cdot \sin \gamma_{0},
$$

and thus $f^{\prime}\left(\gamma_{0}\right) \cdot \sin ^{2}\left(\gamma_{0} / 2\right)>1 / 2\left(\gamma_{0}-3\right)>0$. Consequently $f^{\prime}(\gamma) \sin ^{2}(\gamma / 2)$ and also $f^{\prime}(\gamma)$ are positive in the interval $2 \pi<\gamma<\gamma_{0}$. Hence $f(\gamma)$ is increasing. As $f(\gamma)$ has no lower bound as $\gamma$ approaches $2 \pi$ from above, and

$$
f\left(\gamma_{0}\right)=6-\gamma_{0}^{2} / 2-3 \gamma_{0} \cot \left(\gamma_{0} / 2\right)<-\gamma_{0}^{2} / 2<0,
$$

then it can be concluded that $f(\gamma)<0$ for $2 \pi<\gamma<\gamma_{0}$.

Finally consdier the interval $\gamma_{0}<\gamma<3 \pi$. Since $v(\gamma)>0, f^{\prime}\left(\gamma_{0}\right) \sin ^{2}\left(\gamma_{0} / 2\right)>$ 0 , and thus $f^{\prime}\left(\gamma_{0}\right)>0$, then $f^{\prime}(\gamma)>0$ holds for $\gamma_{0}<\gamma 3 \pi$. Hence $f(\gamma)$ is increasing. But

$$
f(3 \pi)=6-9 \pi^{2} \cdot / 2<0,
$$

so that a $\gamma$ exists such that $f(\gamma)<0$ occurs in the interval.

It was shown in (4.5) that if the image of $|z|=1$ was to be convex for $\gamma_{0}<y<3 \pi$, then $f(y)>0$. Thus the image is not convex for the complete interval, which completes the proof of Theorem 3.

\section{REFERENCES}

1. E. Egerváry, Abbildungseigenschaften der arithmetischen Mittel der geometrischen Reihe, Math. Z. 42 (1936-1937), 221-230. 
2. L. Fejér, Potenzreihen mit mehrfach monotoner Koeffizientenfolge und ihre Legendrepolynome, Proc. Cambridge Philos. Soc. 31 (1935), 307-316.

3. Trigonometrische Reihen und Potenzreihen mit mehrfach monotoner Koeffizientenfolge, Trans. Amer. Math. Soc. 39 (1936), 18-59.

4. - Untersuchungen über Potenzreihen mit mehrfach monotoner Koeffizientenfolge, Acta Sci. Math. Szeged 8 (1936), 90-116.

5. L. Fejér and G.'Szegö, Über die monotone Konvergenz von Potenzreihen mit mehrfach monotoner Koeffizientenfolge, Prace Mat. Fiz. 44 (1935), 15 - 25.

6. _- Special conformal mappings, Duke Math. J. 18 (1951), 535-547.

7. K. Knopp, Mehrfach monotone Zahlenfolgen, Math. Z. 22 (1925), 75 - 85.

8. M. Schweitzer, The partial sums of second order of the geometric series, Duke Math. J. 18 (1951), 527-533.

9. S. Sidon, Über Potenzreihen mit monotoner Koeffizientenfolge, Acta Sci. Math. Szeged 9 (1938-1940), $244-246$.

10. G. Szegö, Power series with multiply monotonic sequences of coefficients, Duke Math. J. 8 (1941), 559-564.

University of California

Santa Barbara College 



\title{
PACIFIC JOURNAL OF MATHEMATICS
}

\section{EDITORS}

\author{
M. M. SCHIFFER* \\ Stanf ord University \\ Stanf ord, California \\ E. HewitT \\ University of Washington \\ Seattle 5, Washington
}

\author{
P. P. DILWCR TH \\ California Institute of Technology \\ Pasadena 4, California \\ E. F. BECKENBACH** \\ University of California \\ Los Angeles 24, California
}

\section{ASSOCIATE EDITORS}

$\begin{array}{ll}\text { H. BUSEMANN } & \text { P. R. HALMOS } \\ \text { HERDERT FF.DERER } & \text { HEINZ HOPF } \\ \text { MARSH ALLL IHALL } & \text { R. D. JAMES }\end{array}$

MARSH AL.L II ALL

\author{
R. D. JAMES
}
BORGE JESSEN
PAUL LÉVY
GEORGE PÓLYA

\author{
J. J. STOKER \\ E. G. STRAUS
}
KÖSAKU YOSIDA

\section{SPONSORS}

UNIVERSITY OF BRITISH COLUMBIA CAI IFORNIA ENSTITUTE OF TECHNOLOGY UNIVERSITY OF CALIFORNIA, BERKELEY UNIVERSITY OF CALIFORNIA, DAVIS UNIVERSITY OF CALIFORNIA, LOS ANGELES UNIVERSITY OF CALIFORNI A, SANTA BARBARA UNIVERSITY OF NEVADA OREGON STATE COLLEGE UNIVERSITY OF OREGON
UNIVERSITY OF SOU THERN CALIFORNIA STANFORD RESE.ARCH INSTITUTE STANFORD UNIVERSITY WASHING TON STATE COLLEGE UNIVERSITY OF WASHINGTON

AMERICAN MATHEMATICAL SOCIETY HUGHES AIRCRAFT COMPANY SHELL DEVELOPMENT COMPANY

* To be succeeded in 1955, by H.L. Royden, Stanford University, Stanford, California.

${ }^{* *}$ To be succeeded in 1955, by E.G. Straus, University of California, Los Angeles 24, Calif.

\author{
Vari-Type Composition by \\ Elaine Barth
}

Printed in the United States of America by

Edwards Brothers, Inc., Ann Arbor, Michigan

UNIVERSITY OF CALIFORNIA PRESS * BERKELEY AND LOS ANGELES COPYRIGHT 1954 BY PACIFIC JOURNAL OF MATHEMATICS 


\section{Pacific Journal of Mathematics}

\section{Vol. 4, No. 1 \\ May, 1954}

Hugh D. Brunk, On the growth of functions having poles or zeros on the positive real axis ................................. 1

J. Copping, Application of a theorem of Pólya to the solution of an infinite

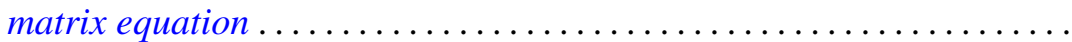

James Richard Jackson, On the existence problem of linear programming ................................... 29

Victor Klee, Invariant extension of linear functionals ............... 37

Shu-Teh Chen Moy, Characterizations of conditional expectation as a transformation on function spaces ..................... 47

Hukukane Nikaidô, On von Neumann's minimax theorem .............. 65

Gordon Marshall Petersen, Methods of summation .................. 73

G. Power, Some perturbed electrostatic fields .................. 79

Murray Harold Protter, The two noncharacteristic problem with data partly

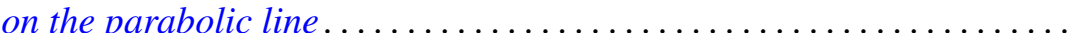

S. E. Rauch, Mapping properties of Cesàro sums of order two of the geometric series........................................... 109

Gerson B. Robison, Invariant integrals over a class of Banach spaces . . . . 123

Richard Steven Varga, Eigenvalues of circulant matrices .............. 151 